\title{
Mit einem iPad länger sicher und unabhängig zu Hause leben
}

Nadja Mueller Schmid

Damit ältere Menschen sicher im eigenen Haushalt leben können, hat der Verein für Altersbetreuung im Oberen Fricktal eine Sicherheitsapplikation für iPads entwickelt. Die Autorin hat das Projekt begleitet und dessen Erfolgsfaktoren identifiziert. Dazu gehört die Erkenntnis, dass für die Etablierung technischer Lösungen soziale Kompetenzen ebenso wichtig sind wie technische.

\section{Vivre chez soi plus longtemps, en sécurité et de} manière autonome avec un $\mathrm{iPad}$

Afin que les personnes âgées puissent vivre en sécurité dans leur propre maison, la Verein für Altersbetreuung im Oberen Fricktal (Association pour la prise en charge des personnes âgées dans le Haut Fricktal, VAOF) a développé une application de sécurité pour iPad. L'auteure a accompagné le projet et identifié ses facteurs de succès dont fait partie la connaissance selon laquelle les compétences sociales sont tout aussi importantes que les compétences techniques pour mettre en place des solutions technologiques.

DOI 10.1024/2297-5160/a000014

\section{Zentrale Leitlinien}

Der Verein für Altersbetreuung im Oberen Fricktal (VAOF) hat Pionierarbeit geleistet und eine mobile Sicherheitslösung für selbständig lebende ältere Menschen entwickelt. Das Pilotprojekt zeigt, welche Kriterien eine technische Sicherheitslösung erfüllen muss, damit sie durch die Zielgruppe angenommen wird.

Seit Beginn lagen dem Projekt vier zentrale Leitlinien zugrunde, die sich aus den Bedürfnissen von Nutzern und Dienstleistern gleichermassen ableiten:

1. Die Technik muss intuitiv verstanden und bedient werden können.

2. Administration und Datenerfassung müssen visuell und haptisch benutzerfreundlich sein.

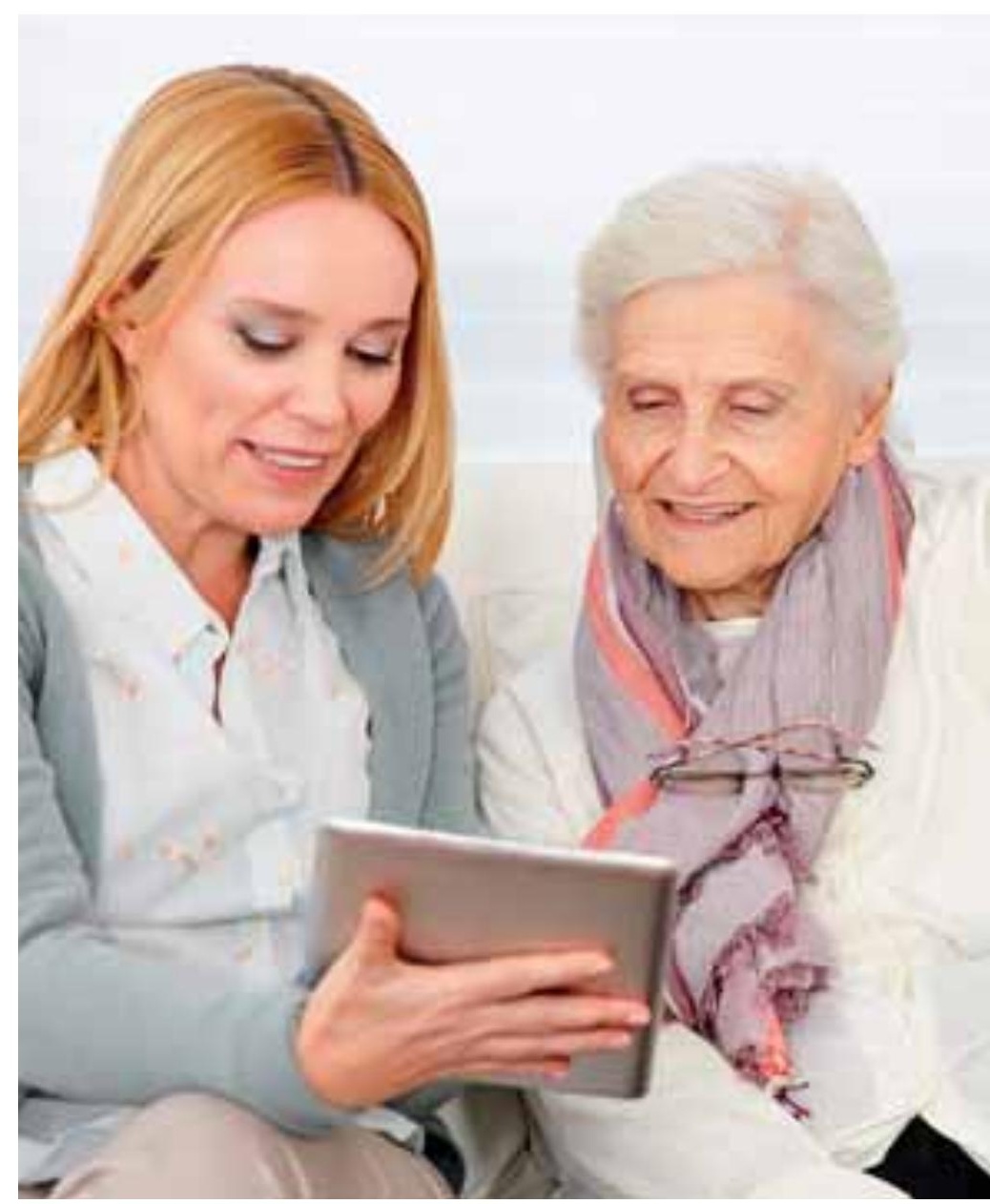

3. Auf sorgfältige Schulung wird besonderes Augenmerk gerichtet, damit Personen, die nicht mit den neuen Medien aufgewachsen sind, die Technik beherrschen können.

4. Das Produkt basiert auf bestehenden technischen Schnittstellen und nutzt eine zukunftsträchtige und ausbaufähige Technologie.

\section{Hemmungen erkennen}

Eine Entwicklerfirma wurde beauftragt, auf Basis dieser Leitlinien den Prototyp einer iPad-Applikation zu entwickeln - die Hisi-App. Als externe Fachperson begleitete die Autorin das Projekt, um die bedürfnisgerechte Umsetzung sicherzustellen. Die Benutzerfreundlichkeit des Prototyps 
wurde mehrmals getestet und danach von den Programmierern angepasst. Ausgewählte Haushalte probierten mit der Fachperson die Hisi-App auf einem iPad aus und gaben im persönlichen Gespräch Feedback. Die Fachperson konnte so auch unbewusste Handlungen der Nutzenden erkennen und damit Schwierigkeiten in der Bedienung aufdecken. Die persönlichen Gespräche enthüllten auch emotionale Hemmungen bei der Verwendung des neuen Mediums: Das iPad wirkte modern und kompliziert. Die Testpersonen konnten sich damals kaum vorstellen, wozu es nützlich sein soll.

\section{Eine simple Sache}

In diesem partizipativen Prozess entstand die «Hisi-App», eine Applikation für iPads mit drei Grundfunktionen und einer Administrationskonsole für die Verwaltung. Die Funktionen werden einfach und intuitiv aktiviert: In der Regel wird auf dem Touchscreen ein Pfeil nach rechts verschoben - eine simple Sache also.

Die drei Funktionen der Hisi-App:

- Mit dem Sicherheitscheck bestätigt man täglich, dass alles gut ist. Passiert etwas und man kann selber keine Hilfe rufen, trifft diese automatisch innert $24 \mathrm{~h}$ ein. Der Projektverlauf zeigt, dass der Sicherheitscheck eine zentrale Funktion ist.

- Die Zusatzfunktion Extra Check wird aktiviert, um sich für kürzere Zeiträume abzusichern, beispielswiese vor dem Duschen, wenn erhöhtes Sturzrisiko besteht.

- Wird der Hilferuf ausgelöst, kommt sofort Hilfe. Diese Funktion wird nur in Notfällen eingesetzt.

Ist der Akku leer oder fehlt die Internetanbindung, funktioniert die Hisi-App nicht. Dann erhält die Zentrale eine automatische Nachricht und erinnert telefonisch daran, das Gerät aufzuladen oder die Internetanbindung zu prüfen - eine wichtige Erinnerungsfunktion für diejenigen, die keine Routinen für mobile Technologien haben.

\section{Komplexes Gesamtsystem}

Für die Nutzenden wirkt die Hisi-App einfach. Das Gesamtsystem ist aber komplex und umfasst weitere Anwendungen, Geräte, Betriebssysteme, Server und keinesfalls zu vernachlässigen: Ein effektives und effizientes Helfersystem für den Ernstfall.

Die Internetanbindung dient der Verknüpfung und Kommunikation innerhalb des Gesamtsystems. Der Alarmserver eines Alterszentrums (Sirio) leitet die Signale der Hisi-App an den zuständigen Empfänger weiter. Der Backend-Server enthält die Administrationskonsole für die Nutzer- und Geräteverwaltung und speichert die gesamte Logik des Systems.

Beim VAOF laufen alle Fäden zusammen: Der Kontakt zu den technischen Zulieferern, die Administration und die Nutzerbetreuung. Die Administrationsplattform wurde durch die Neoos $\mathrm{GmbH}$ parallel zur Entwicklung der Hisi-App entworfen und in enger Zusammenarbeit mit dem VAOF weiterentwickelt und getestet. Die Mitarbeitenden des VAOF mussten sich auf drei Ebenen schulen und organisieren: technisch, administrativ und in betreuerischer Hinsicht. Zusätzlich wurden Prozesse zur Qualitätssicherung definiert.

Im Ernstfall müssen die Mitarbeitenden des Alterszentrums ausrücken. Die Einsatzzentrale wurde durch das VAOF Team für das Vorgehen bei einem Notfall geschult und erhielten eine Dokumentation über den Ablauf bei einer Hisi-Alarmierung.
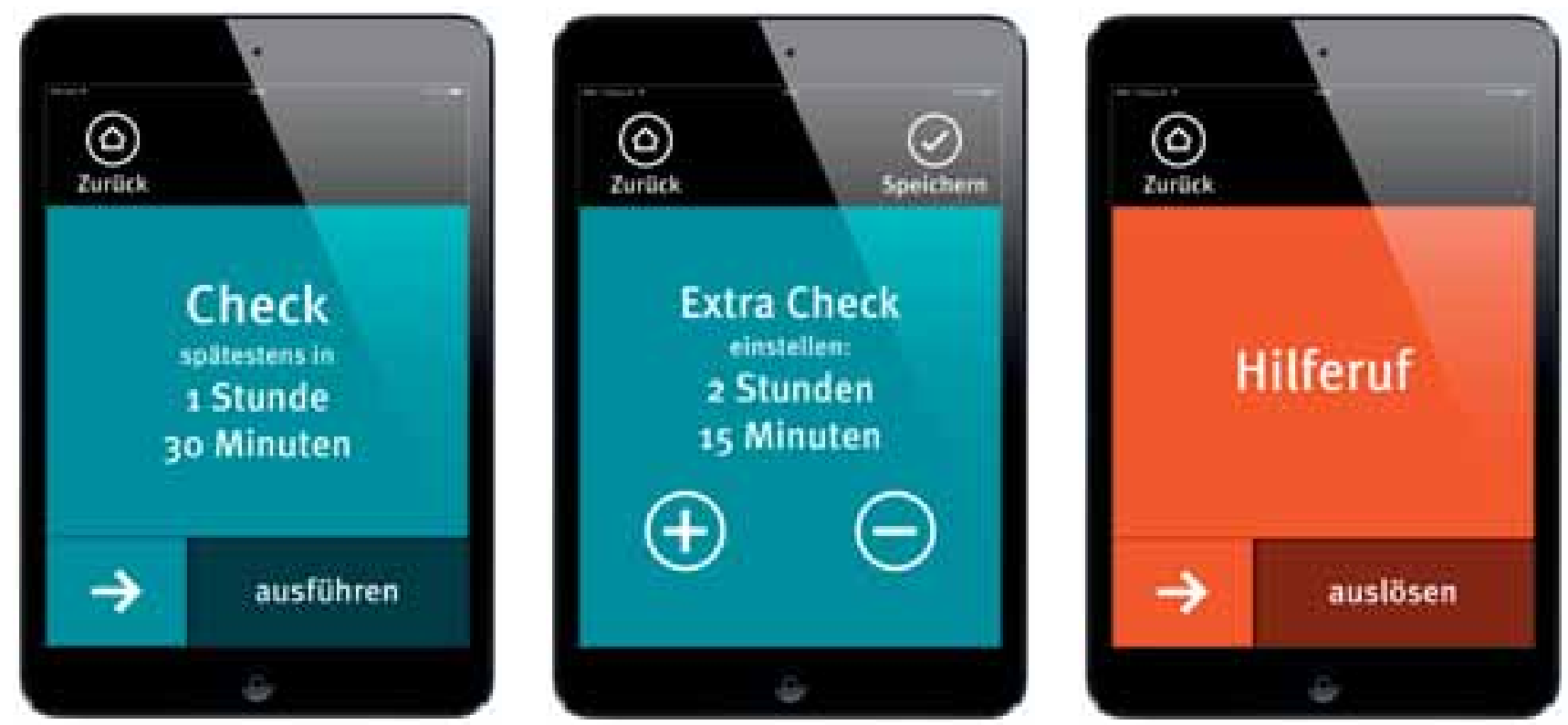

So werden die drei Grundfunktionen auf dem Display eines iPads dargestellt. 


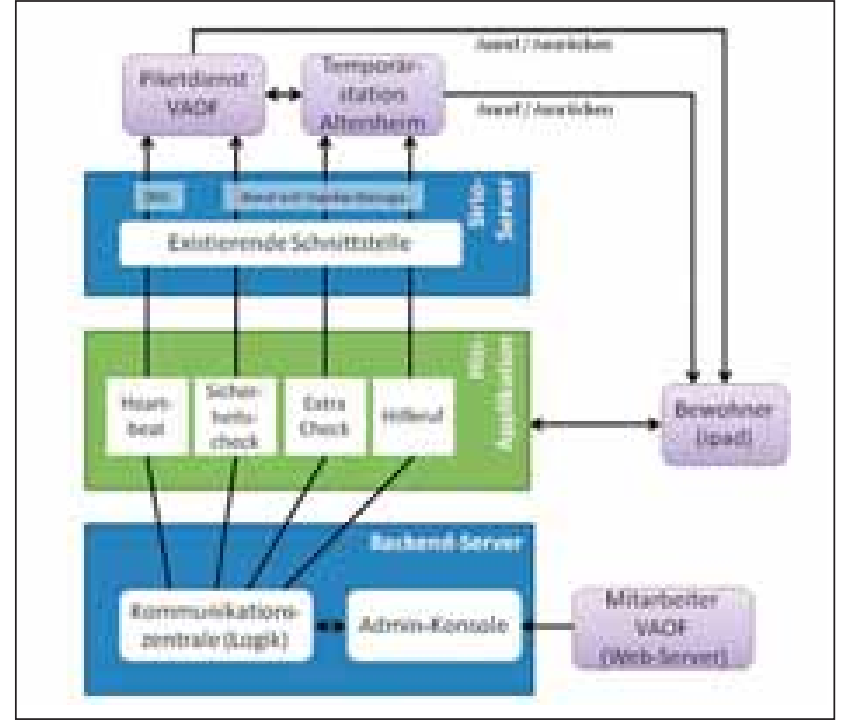

Systemübersicht: Hinter einer einfachen App steht ein komplexes Gesamtsystem (Vereinfachte Darstellung).

\section{Haushalte im Pilot}

Nachdem die App und die Administrationskonsole fertig entwickelt, mit den Servern verknüpft und alle involvierten Stellen geschult waren, wurde das ganze System in seiner realen Umgebung auf Herz und Nieren geprüft: In einer rund sechsmonatigen Pilotphase wurde das iPad in 19 Haushalten installiert. Schulungen erfolgten durch eine Mitarbeiterin des VAOF direkt am Gerät und im eigenen Haushalt. Zudem hat der VAOF eine leicht verständliche Bedienungsanleitung entwickelt. Im weiteren Projektverlauf wurden aber weitere Massnahmen notwendig: Durch die seltene Nutzung der Applikation (nur im Notfall) geht deren Handhabung schnell vergessen. Deshalb führt der VAOF nun monatlich bei allen Haushalten einen Testlauf durch, in dem auch der Ernstfall live geprobt wird. Dadurch wird nicht nur der Wissensstand der Nutzenden aktualisiert, sondern insgesamt auch der Betreuungsaufwand seitens VAOF reduziert.

\section{Attraktive Zusatzfunktionen - hohe Akzeptanz}

Das iPad mit Internetanbindung eröffnet auch neue Möglichkeiten, die nicht mit der «Hisi-App» zusammenhängen: E-Mail, Kommunikation mit den Enkeln, Vernetzung mit der Wandergruppe, Video-Telefonie mit Verwandten im In- und Ausland sowie unkomplizierte Informationsbeschaffung: Fahrplan, Wetter, Nachrichten etc. Wie das Pilotprojekt zeigt, sind es gerade diese attraktiven Zusatzfunktionen, die treibend für die Nutzung der Hisi-App und des iPads wirken.

Diese Anwendungsmöglichkeiten haben auch bei den Bewohnenden des Alterszentrums Neugier geweckt. Der VAOF musste aus ökonomischen Gründen den Support für sein Hilferufsystem von jenem für allgemeine Fragen zum
Gerät und weiteren Nutzungsmöglichkeiten trennen. Eine technisch versierte Bewohnerin hat diese Supportfunktion auf freiwilliger Basis übernommen, was die Akzeptanz der Nutzung des Gerätes bei den Bewohnenden noch erhöhte.

\section{Die Quintessenz}

Das komplexe System, in das die Hisi-App eingebettet ist, wurde in rund einem Jahr aufgebaut. Aus den Erfahrungen lassen sich allgemeine Kriterien für die erfolgreiche Umsetzung technischer Sicherheitssysteme ableiten:

1. Neben finanziellen Ressourcen und technischer Infrastruktur sind eine sorgfältige Vorbereitung und die enge Zusammenarbeit zwischen allen Beteiligten essenziell. Die Bedürfnisse und Möglichkeiten aller Ansprechgruppen müssen bereits beim Projektstart evaluiert und während der Entwicklungsphase eingebracht werden.

2. Die Programmierung und Anpassung der Frontendund Backend-Schnittstellen stellt fachliche Anforderungen an die Zulieferer wie auch an die Projektleitung mit nicht zu unterschätzendem Zeitaufwand.

3. Bei Projektleitung und Verwaltung braucht es neben technischen auch soziale Kompetenzen zur Betreuung der technisch weniger affinen Nutzenden. Eine Aufteilung der beiden Aufgabenbereiche ist aufgrund der vielen Überschneidungen nicht sinnvoll.

4. Eine technische Lösung wird durch ihre Zielgruppe eher akzeptiert, wenn die Hemmschwellen zur Nutzung von Beginn niedrig gehalten werden: Einfachheit und Benutzerfreundlichkeit des Produktes sowie wiederkehrende und feinfühlige Schulung vor Ort bilden das Fundament für den Erfolg.

5. Nicht zuletzt ist ein technisch ausgefeiltes und bedürfnisorientiert entwickeltes Produkt aber wertlos ohne ein professionelles Helfersystem, das im Bedarfsfall effektiv und effizient reagiert.

\section{Literatur}

Die Entwicklung der Hisi-App war ein Teil eines regionalplanerischen Projekts zum Aufbau einer integrierten Altersversorgung in der Region Fricktal.

Zimmerli, J. et al. (2015). Wohnen im Alter im Fricktal: Planen in der Region. Evaluation regionalplanerischer Massnahmen des Vereins für Altersbetreuung im Oberen Fricktal (VAOF), 2012-2014. Schlussbericht unter: http://www.age-stiftung.ch/vaof.

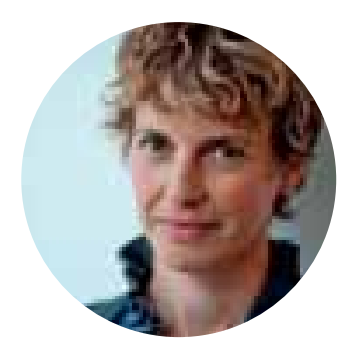

Nadja Mueller Schmid ist Geschäftsleiterin des Marktforschungsinstituts «mfg mueller GmbH» in Zürich. Sie hat im Auftrag des VAOF die Bedürfnisse für eine mobile technische Lösung eruiert und die Umsetzung des Projektes begleitet.

www.mfgmueller.ch 